molecules is currently one of the leading causes of allergic asthma and rhinitis in the workplace.

Objectives Describe the socio-professional, clinical, functional and allergological characteristics of cases of asthma allergic to isocyanates, and assess their impact on medical fitness for work.

Methods Retrospective study covering all cases of occupational allergic asthma and rhinitis to isocyanates collected from the Department of Occupational Medicine of La Rabta hospital during the period from January 2000 to July 2020.

Result: This is a series of 26 patients with a mean age of $42.62 \pm 9.01$ years, including 19 women and 7 men. The majority belonged to the automotive sector (19 cases). They were mainly workers responsible for gluing and sheathing the steering wheels (16 cases). The average seniority in the post was $12.88 \pm 6.01$ years. The lack of personal respiratory protection was reported by the majority of patients (24 cases). The first functional manifestations appeared on average 8.15 \pm 5.13 years after exposure, dominated by nasal pruritus and rhinorrhea (16 cases) and wheezing dyspnea (5 cases).Rhythmicity with work was reported by all patients. The physical examination revealed a congestive mucosa (3 cases) with severe obstruction (1 case). Reversible obstructive or airway hyper reactivity was confirmed in 11 cases. Finally, we identified 15 cases of allergic rhinitis, 7 cases of allergic asthma and 4 cases of rhinitis associated with asthma. This morbidity justified an adaptation of the workstation in 12 patients and a mutation of the post in 12 cases. the majority of patients (23 cases) benefited from a declaration of this morbidity as occupational disease.

Conclusion Occupational exposure to isocyanates represents a significant allergic risk. This risk remains avoidable by putting in place appropriate individual and collective preventive measures.

\section{RF-441 RESPIRATORY AND ALLERGIC SYMPTOMS IN CANNABIS CULTIVATION WORKERS, CALIFORNIA, USA.}

${ }^{1}$ Stella Beckman, Chelsea Langer, Marc Schenker. 'University of California - Davis, United States

\subsection{6/OEM-2021-EPI.385}

Introduction Cannabis (Cannabis sativa, marijuana) is the largest cash crop in California. While it is difficult to characterize the size of the industry, total production is estimated at 15 to 16 million pounds per year with total revenues exceeding $\$ 10$ billion per year. There are approximately 40,000 full-time equivalent cannabis jobs in the state. Despite the huge size and rapid growth of the industry, there has been little research on the health and safety of cannabis workers.

Objectives The goal of this pilot study was to explore the feasibility of collecting cannabis worker health data with a selfadministered survey and to analyze the first systematically collected data on occupational respiratory and allergic symptoms among California cannabis cultivation workers.

Methods We performed a cross-sectional survey of 29 workers at two cannabis farms in the Davis/Sacramento, California area. The questionnaire elicited information about the participants' personal and job characteristics and nasal, respiratory, dermal, and eye symptoms.
Results All eligible workers chose to participate. Most participants were men age $\leq 30$ years, with $48 \%$ of participants self-identifying as non-Hispanic white and 58\% reporting at least some college education. The median tenure of all work in the cannabis industry was 26 months (IQR: 9, 78). All participants were current or past cannabis users. Five (17\%) workers reported physician-diagnosed asthma; however, 11 workers (38\%) reported symptoms suggestive of asthma. Work-related symptoms of any type were reported by 11 (38\%) participants.

Conclusion The survey was a satisfactory data collection method. The participants had a demographic makeup distinct from traditional California agricultural workers, who are predominantly Latino, speak Spanish, and have less formal education. The high proportion of respondents with symptoms suggestive of asthma raises concern; sensitization to the cannabis plant has been reported among cannabis users and there are many well-documented agricultural exposures causing asthma and asthma-like syndrome.

\section{RF-14 THE ASSOCIATION BETWEEN SHIFT WORK, MENTAL HEALTH AND CARDIOMETABOLIC HEALTH IN THE ATLANTIC PATH COHORT}

${ }^{1}$ Ellen Sweeney, Zhijie Michael Yu, Trevor Dummer, Yunsong Cui, Vanessa DeClercq, Cynthia Forbes, Scott A Grandy, Melanie Keats, Anil Adisesh. ${ }^{1}$ Dalhousie University, Canada

\subsection{6/OEM-2021-EPI.386}

Introduction Contemporary work environments increasingly rely upon a 24-hour work cycle resulting in more employees exposed to shift work. Thirty percent of working age Canadians work evening, night and rotating shifts. Compared to regular daytime work, shift work has the potential for disturbing sleep patterns and disrupting circadian rhythms with adverse health effects.

Methods A population health study was conducted with 4,155 shift workers and 8,258 non-shift workers from the Atlantic PATH cohort. Linear and logistic regression models were used to assess the differences in i) self-reported mental health measures between shift workers and non-shift workers and ii) anthropometric measures (body adiposity) and self-reported cardiometabolic disease outcomes (obesity, diabetes, and cardiovascular disease).

Results Shift workers reported higher levels of each of the mental health domains. There was a significant increased risk of depression $(\mathrm{OR}=1.13,95 \% \mathrm{CI}, 1.00-1.27)$ and poor selfrated health $(\mathrm{OR}=1.13,95 \% \mathrm{CI}, 1.14-1.55)$ among shift workers compared to non-shift workers. There was a significant increased risk of cardiovascular disease, obesity, and diabetes among shift workers despite higher levels of physical activity and lower levels of sedentary behaviour compared to matched controls. Shift workers were $17 \%$ more likely to be obese (95\% CI 7-27) and 27\% more likely to have diabetes (95\% CI 8-51).

Conclusions Shift work is associated with cardiometabolic health and mental health, as well as depression. The association between shift work and cardiometabolic health was independent of body mass index for cardiovascular disease and diabetes, and independent of fat mass index for diabetes. Subsequently, shift workers have an increased risk of developing other chronic disease. 